\title{
Obstrucción intestinal secundaria a íleo biliar: reporte de caso
}

\author{
Intestinal obstruction secundary to gallstone ileus: case report
}

Jorge A. Morales-Ortiz*, Manuel M. Cota-Novoa, Germán F. Bernal Mora, Xavier E. Salinas-Quintero y

Alba E. González-García

Departamento de Cirugía General, Hospital General Norberto Treviño Zapata de Cd. Victoria, Tamaulipas, México

\begin{abstract}
Resumen
El íleo biliar es una obstrucción intestinal secundaria a la presencia de cálculos biliares en el intestino delgado. Es una complicación rara de la colelitiasis y representa el 1-3\% de las causas de obstrucción intestinal. El diagnóstico es difícil dado que la clínica es inespecífica, con un cuadro de oclusión intestinal intermitente; la tríada de Rigler (neumobilia, lito y distensión abdominal) es patognomónica. Dentro de los auxiliares diagnósticos se encuentran la radiografía de abdomen, el ultrasonido y la tomografía computarizada abdominal. El tratamiento debe individualizarse dependiendo de las condiciones del paciente, siendo de elección la laparotomía con enterotomía.
\end{abstract}

Palabras clave: Íleo biliar. Obstrucción intestinal. Colelitiasis. Laparotomía.

\begin{abstract}
Gallstone ileus is an intestinal obstruction that is secondary to the presence of gallstones in the small intestine. It is a rare complication of cholelithiasis, which represents $1-3 \%$ of the causes of intestinal obstruction. The diagnosis is difficult given that the symptoms are nonspecific with intermittent intestinal obstruction, Rigler's triad (pneumobilia, stone and abdominal distention) is pathognomonic. Among the diagnostic aids are abdominal radiography, ultrasound and abdominal tomography. Treatment should be individualized depending on the patient's conditions, with laparotomy with enterotomy being the treatment of choice.
\end{abstract}

Key words: Gallstone ileus. Intestinal obstruction. Cholelithiasis. Laparotomy.

\section{Introducción}

El íleo biliar, descrito por Bartholin en 1654 en una necropsia, se define como una obstrucción mecánica secundaria a la presencia de un cálculo biliar en el tracto gastrointestinal-4. Es una complicación de la colelitiasis y una causa rara de obstrucción intestinal (1-3\%), pudiendo llegar hasta el $25 \%$ en pacientes mayores de 65 años ${ }^{2,3}$, producida por el paso de un cálculo biliar a través de una fistula entre la vesícula y el duodeno. Hasta el $30 \%$ de la población adulta tiene colelitiasis, pero solo un $0.3-0.5 \%$ de los pacientes con colelitiasis desarrollarán íleo biliar.

\section{Correspondencia:}

*Jorge A. Morales-Ortiz

Prol. Pedro José Méndez 2008

Loma del Gallo

Fecha de recepción: 09-01-2021

C.P. 89460 , Ciudad Madero, Tamaulipas, México

E-mail: jm.91@ @live.com.mx
Cir Cir. 2021;89(S2):31-33

Contents available at PubMed www.cirugiaycirujanos.com

0009-7411/@ 2021 Academia Mexicana de Cirugía. Publicado por Permanyer. Este es un artículo open access bajo la licencia CC BY-NC-ND (http://creativecommons.org/licenses/by-nc-nd/4.0/). 
El $90 \%$ de los cálculos obstructivos son mayores de $2 \mathrm{~cm}$ de diámetro y la mayoría mide más de 2.5 $\mathrm{cm}^{5}$. Del $50 \%$ al $70 \%$ de los cálculos biliares se impactan en el íleon, que es el segmento más angosto del intestino, seguido del yeyuno (20-40\%), el estómago y el duodeno $(10 \%)^{2}$. La obstrucción colónica es rara, ya que el diámetro normal del colon generalmente permite el paso de los cálculos biliares.

El diagnóstico resulta difícil dado que la clínica es inespecífica. Existen tres formas de presentación clínica: aguda, que corresponde a la clásica presentación de íleo biliar; subaguda, como oclusión parcial intestinal; y crónica, conocida como síndrome de Karewsky, caracterizada por episodios repetidos del dolor que remiten conforme pasa el lito al intestino ${ }^{3}$.

Dentro de los auxiliares diagnósticos, la radiografía de abdomen tiene un $43 \%$ de sensibilidad. La tríada de Rigler (neumobilia, lito y distensión de asas intestinales) es patognomónica ${ }^{3}$, pero se presenta en menos del $50 \%$ de los casos. El ultrasonido tiene una sensibilidad del $78 \%$, mientras que la tomografía computarizada contrastada posee una sensibilidad del $93 \%$ y una especificidad cercana al $100 \%$, por lo cual se considera el método de referencia ${ }^{5,6}$. El diagnóstico preoperatorio del presente caso fue posible mediante tomografía computarizada (Fig. 1).

\section{Caso clínico}

Varón de 56 años con antecedente de diabetes mellitus tipo 2 de 2 años de diagnóstico y consumo de alcohol ocasional. Inició en los días previos con dolor de tipo cólico en el epigastrio y el hipocondrio derecho, acompañado de náusea y vómito de contenido gastroalimentario, con intolerancia a la vía oral. Fue manejado con analgésicos, sin mejoría, y acudió al servicio de urgencias por presentar vómito, dolor abdominal intenso y distensión, refiriendo la última evacuación 6 días antes. Se inició manejo sintomático con analgésicos y colocación de sonda nasogástrica, y se solicitaron paraclínicos.

En la exploración física el paciente se encontraba estable, con abdomen distendido, peristalsis ausente, dolor a la palpación media y profunda en el hipocondrio derecho, Blumberg positivo, y sin presencia de materia fecal en el tacto rectal.

Los parámetros de laboratorio fueron: hemoglobina $14.3 \mathrm{~g} / \mathrm{dl}$, plaquetas 294,000 , leucocitos 17.3 , neutrófilos $76 \%$, Na 136, K 3.5, Cl 100, glucosa 176, urea 32 , creatinina 0.4 , bilirrubina total (BT) 0.91 , bilirrubina directa $(\mathrm{BD})$ 0.10, bilirrubina indirecta $(\mathrm{BI}) \quad 0.80$,

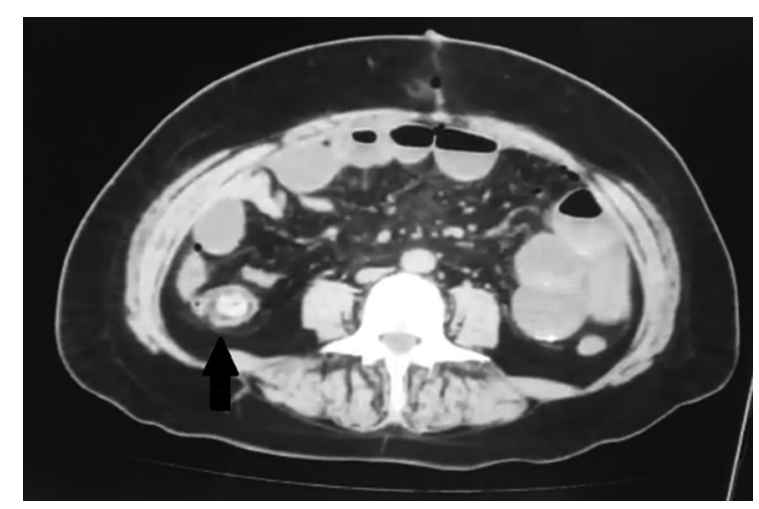

Figura 1. Tomografía computarizada abdominal que muestra una imagen hiperdensa en la fosa ilíaca derecha en relación con litiasis ectópica (flecha).

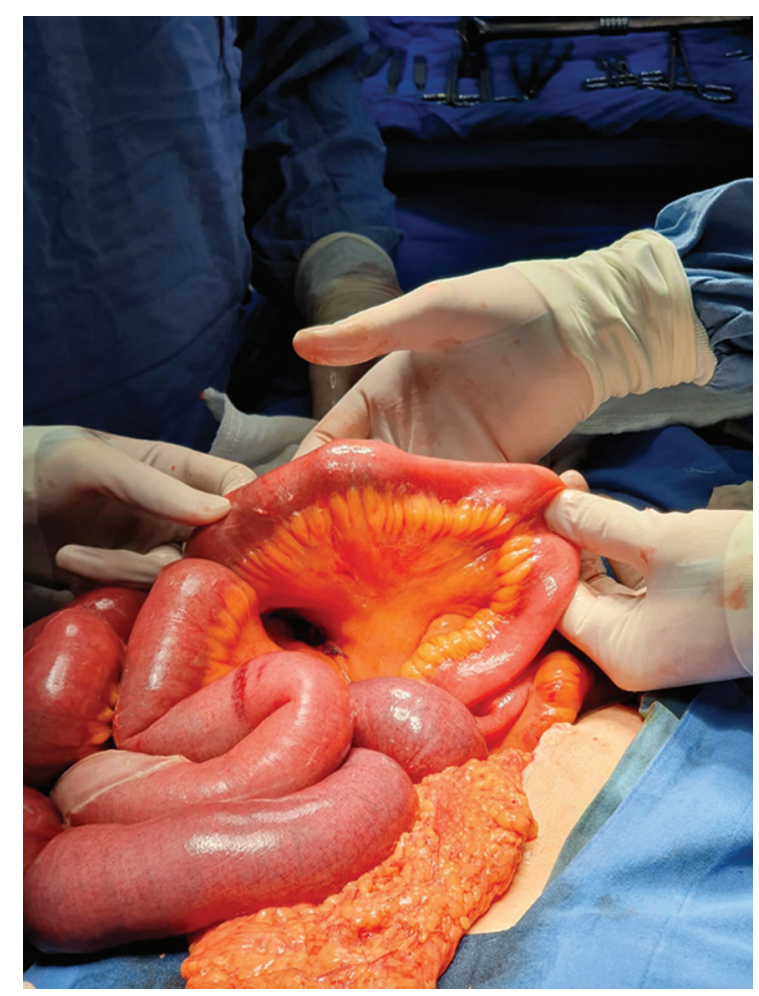

Figura 2. Lito impactado en el íleon.

fosfatasa alcalina 110 , lactato deshidrogenasa 343 , albúmina 3.5, lipasa 157, TP 13.4, TPT 25.1 e International Normalized Ratio (INR) 1.05.

La tomografía computarizada abdominal mostró una imagen hiperdensa en la fosa ilíaca derecha, sin líquido libre ni aire libre en la cavidad.

Se realizó una laparotomía exploradora que reveló una fístula colecistoduodenal y un lito impactado en el íleon (Fig. 2), por lo que se decidió realizar una 


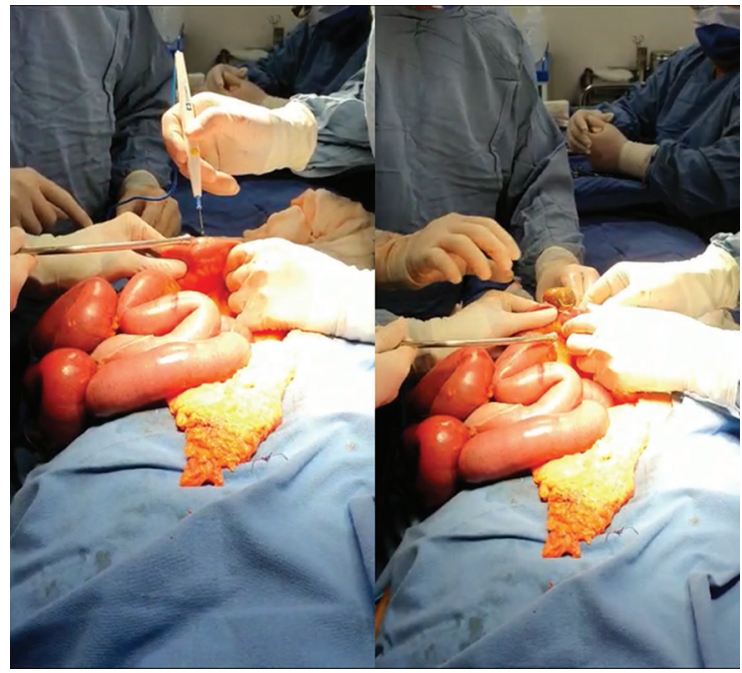

Figura 3. Enterotomía.

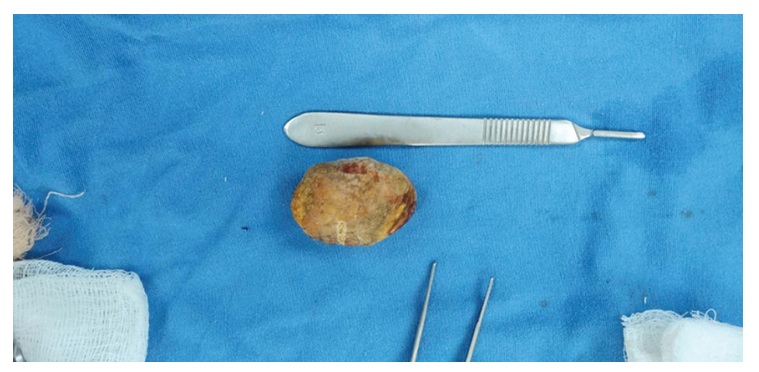

Figura 4. Lito de $6 \times 6 \mathrm{~cm}$.

enterotomía (Fig. 3) a $90 \mathrm{~cm}$ del ángulo de Treitz para la extracción del lito de $6 \times 6 \mathrm{~cm}$ (Fig. 4), sin complicaciones.

\section{Discusión}

El íleo biliar es una causa sumamente infrecuente de obstrucción intestinal; sin embargo, la tasa de mortalidad por íleo biliar sigue siendo alta, del $4.5 \%$ al $25 \%$, debido a que hasta en el $50 \%$ de los casos el diagnóstico se realiza durante una laparotomía exploradora ${ }^{4,7}$.

La intervención quirúrgica mediante enterotomía es el tratamiento de elección en los pacientes con íleo biliar, por su menor morbimortalidad en comparación con otras técnicas. Se sugiere realizar el manejo en dos fases: enterolitotomía seguida de colecistectomía y cierre de la fístula en 4 a 6 semanas $^{4}$. Sin embargo, se prefiere la enterolitotomía, ya que el $50 \%$ de las fístulas cierran de forma espontánea.
Al igual que con cualquier otra afección quirúrgica aguda, la atención debe centrarse primero en el estado general del paciente y el tratamiento médico de cualquier obstrucción intestinal ${ }^{7}$, con reanimación hidroelectrolítica estricta al ingreso, así como realización de tomografía computarizada abdominal. La agudeza del paciente dicta el momento de la operación; una vez que se realiza el diagnóstico, se indica una pronta exploración.

\section{Agradecimientos}

Los autores agradecen al personal de urgencias y de quirófano del Hospital General Norberto Treviño Zapata de Cd. Victoria, Tamaulipas, México.

\section{Responsabilidades éticas}

Protección de personas y animales. Los autores declaran que para esta investigación no se han realizado experimentos en seres humanos ni en animales.

Confidencialidad de los datos. Los autores declaran que han seguido los protocolos de su centro de trabajo sobre la publicación de datos de pacientes.

Derecho a la privacidad y consentimiento informado. Los autores han obtenido el consentimiento informado de los pacientes y/o sujetos referidos en el artículo. Este documento obra en poder del autor de correspondencia.

\section{Conflicto de intereses}

Los autores declaran que no existe ningún conflicto de intereses en la realización de este trabajo.

\section{Bibliografía}

1. Ploneda Valencia CF, Gallo Morales M, Rinchon C, Navarro Muñiz E, Bautista López CA, De la Cerda Trujillo LF, et al. El íleo biliar: una revisión de la literatura médica. Rev Gastroenterol Mex. 2017;82:248-254.

2. Toral-Chan AI, Palacios-Padrón A, Vázquez-Hernández R. Íleo biliar: un reto diagnóstico y terapéutico. Rev Hosp Jua Mex. 2019;86:92-5.

3. Sánchez-Pérez E, Álvarez-Álvarez S, Madrigal-Téllez M, Gutiérrez Uvalle G, Ramírez-Velásquez J, Hurtado-López L. Íleo biliar, experiencia en el Hospital General de México Dr. Eduardo Liceaga. Cir Cir. 2017;85:114-20.

4. Martín-Pérez J, Delgado-Plasencia L, Bravo-Gutiérrez A Lorenzo Rocha N, Burillo-Putze G, Medina-Arana V. Enterolitotomía más colecistectomía precoz, una cirugía de control de daños para pacientes con íleo biliar. Cir Cir. 2015;83:156-60.

5. De Monti M, Cestaro G, Alkayyali S, Galafassi J. Gallstone ileus: a possible cause of bowel obstruction in the elderly population. Int J Surg Case Rep. 2018;43:18-20.

6. Gijón de la Santa L, Camarero-Miguel A, Pérez-Retortillo J, Ramia-Ángel J. Síndrome de Bouveret con TC multidetector. Rev Esp Enferm Digest. 2014;106:283-4.

7. Salazar-Jiménez M, Alvarado-Durán J, Fermín-Contreras M, Rivero-Yáñez F, Lupian-Angulo A, Herrera-González A. Íleo biliar, revisión del manejo quirúrgico. Cir Cir. 2018;86:182-6. 